\title{
An Efficient Approach for Accelerating Bucket Elimination on GPUs
}

\author{
Filippo Bistaffa ${ }^{*}$, Nicola Bombieri ${ }^{\dagger}$ and Alessandro Farinelli ${ }^{\ddagger}$ \\ Department of Computer Science, University of Verona, Italy \\ Email: *filippo.bistaffa@univr.it, †nicola.bombieri@univr.it, ${ }^{\dagger}$ alessandro.farinelli@univr.it
}

\begin{abstract}
Bucket Elimination (BE) is a framework that encompasses several algorithms, including Belief Propagation (BP) and variable elimination for Constraint Optimisation Problems (COPs). BE has significant computational requirements that can be addressed by using GPUs to parallelise its fundamental operations, i.e., composition and marginalisation, which operate on functions represented by large tables. We propose a novel approach to parallelise these operations with GPUs, which optimises the table layout so to achieve better performance in terms of increased speedup and scalability. Our approach allows us to process incomplete tables (i.e., tables with some missing variables assignments), which often occur in several practical applications (such as the ones we consider in our dataset). Finally, we can process tables that are larger than the GPU memory. Our approach outperforms the state-of-the-art technique to parallelise BP on GPUs, achieving better speedups (up to $+\mathbf{4 6 6 \%}$ w.r.t. such parallel technique). We test our method on a publicly available COP dataset, measuring a speedup up to $696.02 \times$ w.r.t. the sequential version. The ability of our technique to process large tables is crucial in this scenario, in which most of the instances generate tables larger than the GPU memory, and hence they cannot be solved with previous GPU techniques related to BE.
\end{abstract}

Index Terms-GPU, Bucket Elimination, Constraint Optimisation Problem, Belief Propagation, Junction Tree.

\section{INTRODUCTION}

D YNAMIC Programming (DP) [1] is a well-known method used to solve complex problems by exploiting their optimal substructure property, i.e., the possibility of efficiently decomposing the original problem into smaller subproblems and then construct the global optimal solution.

Several popular DP algorithms used in many different fields have been shown to fall under a general framework called Bucket Elimination (BE) [2]. Some examples are Fourier and Gaussian elimination for linear equalities and inequalities [3], adaptive consistency for Constraint Satisfaction Problems (CSPs) [4], directional resolution for propositional satisfiability [5], Belief Propagation (BP) [6], DP for combinatorial optimisation [7], variable elimination for Constraint Optimisation Problems (COPs) [2] and Distributed COPs (DCOPs) [8]. More precisely, $\mathrm{BE}$ operates by propagating messages over a series of buckets, i.e., sets of constraints (or functions) that depend on a given variable. Such messages, encoded by functions in tabular form, are constructed by means of two fundamental operators, $\oplus$ and $\Downarrow$, often referred as composition and marginalisation. One of the main advantages of $\mathrm{BE}$ is its generality, as such an algorithm can be applied to solve prob- lems in several different fields by changing the fundamental mathematical operations ${ }^{1}$ used by these two operators.

While the number of exchanged messages is usually rather small (i.e., equal to the number of input variables minus one), the computation of the messages themselves represents the most computationally intensive task of the entire algorithm, whose complexity can be precisely predicted and it is exponential w.r.t. a single parameter called induced width of the graph representing the initial problem [2]. For this reason, having a very efficient algorithm to perform such a computation is of utmost importance when dealing with realistic problems, which often require $\mathrm{BE}$ to exchange very large messages (i.e., up to $60 \mathrm{~GB}$ in our experiments).

In recent years, Graphics Processing Units (GPUs) have been successfully used to speedup the computation in different cybernetic applications that feature a high level of parallelism, achieving performance improvements of several orders of magnitude [10] in fields including computer vision [11], human-computer interaction [12], and artificial intelligence [13]. In this work, we are interested in developing a high-performance GPU framework that allows us to deal with the computational effort inherent in the message passing phase of several BE-based algorithms, so to favour its integration in the development of cybernetic solutions, especially in fields like BP, decision making, and scheduling [14]. To this end, our main objective is to devise a solution that fulfils three key requirements. First, since BE is a general algorithm that can be applied to several problems, our framework should be likewise general to allow a wide adoption among different domains, i.e., BP and COPs. Second, our approach should be able to achieve a high computational throughput, by means of optimised memory accesses to avoid bandwidth bottlenecks, a careful loadbalancing to fully exploit the available computational power, and the adoption of well-known parallel primitives [15], [16] to reduce the CPU workload to the minimum. Third, our solution should tackle large-scale real-world problems, and, hence, it should not be limited by the amount of GPU global memory.

In this context, the work of Zheng, Mengshoel, and Chong [17] (later improved by Zheng and Mengshoel [18]) represents, to the best of our knowledge, the only approach that tackles the high degree of parallelism of the BE composition and marginalisation operators (specifically devised for BP) using GPUs. Specifically, they exploit the fact that such opera-

\footnotetext{
${ }^{1} \mathrm{BE}$ for COPs uses + as composition and max as marginalisation. Formally, the mathematical concept that describes these operations is the commutative semiring, discussed in detail by Aji and McEliece [9].
} 
tors execute the same mathematical operation over a large amount of input data and, therefore, they are suitable for the Single Instruction Multiple Data (SIMD) model adopted by these architectures [10]. However, this approach has several drawbacks that hinder its applicability in general real-world scenarios. In fact, it accesses input data in a non-regular way by means of an indexing table, which introduces additional overhead and causes the lack of coalescence and data locality in memory transfers. Furthermore, as a consequence of this lack of regularity, input tables must fit entirely into the GPU global memory, since threads may need to access data that could be anywhere in such tables. Therefore, this method is not applicable to instances whose tables exceed this limitation. Finally, since this approach has been specifically devised for BP, where tables are complete (i.e., they include a row for every possible assignment of the variables in their scope), it is unable to cope with real-world problems with incomplete tables, e.g., COPs, Weighted CSPs (WCSPs) [14] and GraphConstrained Coalition Formation [19], where some variable assignments are unfeasible, i.e., they violate some sort of hard constraint. Notice that, even if it is possible to model incomplete tables as complete ones by representing unfeasible assignments as explicit rows with special values (i.e., $-\infty$ for a maximisation task), this approach is not practical since it results in tables whose size is not manageable.

Against this background, we propose a novel GPU parallel approach for the BE message passing phase that achieves the objectives set above. In more detail, we advance the state-ofthe-art in the following ways:

1) We propose an algorithm to preprocess input tables by organising the columns in a specified order, thus achieving full memory coalesced accesses in the message passing phases. Such preprocessing phase can cope with both complete tables and incomplete ones, and can be realised using fully parallelisable operations. We formally analyse such algorithm proving its correctness and giving the worst case computational complexity.

2) We propose an implementation of the GPU kernel that exploits the table layout specified before. We show that such an arrangement enables pipelined data transfers from the host to the GPU (hence optimising the transfer time) and it allows the use of highly efficient routines for crucial parts of the BE message passing phase (i.e., $\oplus$ and $\Downarrow$ operators). Our method is not limited by the amount of GPU memory, as our data layout allows us to process large tables by splitting them into manageable chunks that meet the memory capabilities of the GPU.

3) We empirically evaluate our approach on two particular $\mathrm{BE}$ realisations, i.e., $\mathrm{BP}$ and $\mathrm{COP}$, adopting standard datasets in both cases. On the one hand, we compare our approach against the one proposed by Zheng and Mengshoel [18] on the same dataset. Our results show significant improvements, by achieving speedups at least $56 \%$ higher than the alternative method (reaching peaks of $+466 \%$ ). On the other hand, we employ our method to solve standard, publicly available WCSPs datasets [14], by modelling them as COPs and then by adopting the
$\mathrm{BE}$ algorithm to compute their solution. The tests show that our method results in a speedup of $696 \times$ w.r.t. the serial approach. We prove the importance of handling tables that exceed the GPU global memory in this scenario, which would otherwise be unsolvable given the dimension of the instances.

\section{BACKGROUND}

The purpose of this section is threefold. First, in Section II-A, we define the theoretical concepts and the algorithms related to BE and BP on JTs. An in-depth discussion of such algorithms can be found respectively in [2] and [6]. Second, Section II-B outlines the features of GPU architectures, used to implement our highly-parallel approach. Finally, Section II-C discusses previous works related to BE on GPUs.

\section{A. Bucket Elimination}

Bucket Elimination (BE) [2] is a unifying algorithmic framework, which generalises DP to accommodate algorithms for many complex problem-solving and reasoning techniques. BE usually accepts inputs in the form of a knowledge-base theory and a query encoded by several functions or relations over subsets of variables (e.g., clauses for propositional satisfiability, constraints, or conditional probability matrices for belief networks). In this paper, we will focus on the resolution of Constraint Networks (CNs), which consist of a set $X=\left\{x_{1}, \ldots, x_{n}\right\}$ of $n$ variables such that $x_{1} \in D_{1}, \ldots$, $x_{n} \in D_{n}$, where $D_{i}$ represents the domain of the variable $x_{i}$, together with a set of $m$ constraints $\left\{C_{1}, \ldots, C_{m}\right\}$, denoting the variables simultaneous legal assignments. Non-legal assignments are denoted as unfeasible. In this article, we are interested in the version of $\mathrm{BE}$ that computes the optimal solution for COPs (Algorithm 1) [2]. COPs can model several realistic problems [8] such as WCSPs [14].

Definition 1: A Constraint Optimisation Problem (COP) is a $\mathrm{CN}$ augmented with a set of functions. Let $F_{1}, \ldots, F_{l}$ be $l$ real-valued functional components defined over the scopes $Q_{1}, \ldots, Q_{l}, Q_{i} \subseteq X$, let $\bar{a}=\left(a_{1}, \ldots, a_{n}\right)$ be an assignment

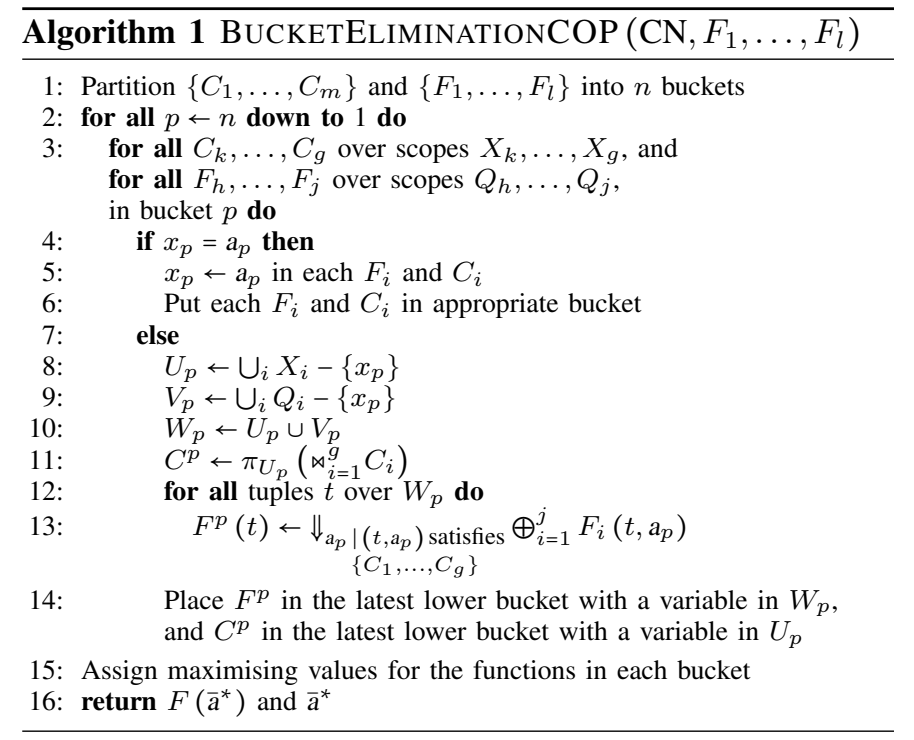


of the variables, where $a_{i} \in D_{i}$. The global cost function $F$ is defined by $F(\bar{a})=\sum_{i=1}^{l} F_{i}(\bar{a})$, where $F_{i}(\bar{a})$ means $F_{i}$ applied to the assignments in $\bar{a}$ restricted to the scope of $F_{i}$. Solving the COP requires to find $\bar{a}^{*}=\left(a_{1}^{*}, \ldots, a_{n}^{*}\right)$, satisfying all the constraints, such that $F\left(\bar{a}^{*}\right)=\max _{\bar{a}} F(\bar{a})$ (or $F\left(\bar{a}^{*}\right)=\min _{\bar{a}} F(\bar{a})$, in case of a minimisation problem).

In Algorithm 1 the $\oplus$ operator is instantiated in Lines 11 and 13 with the relational join and the join sum operation respectively. Moreover, these lines instantiate the $\Downarrow$ operator with the project and the maximisation operations respectively. This difference is due to the fact that constraints are relations [2], as they contain one row per legit assignment of their input variables (while excluding the unfeasible ones). On the other hand, a cost function $F_{i}$ also specifies a value for each row, representing the cost of that particular variable assignment. In the context of the scope of this article, it is crucial to note that the presence of constraints in all these problems inherently makes some variable assignments unfeasible. As a consequence, all the rows in the exchanged messages corresponding to such assignments can be dropped, greatly reducing the memory requirements of the algorithm. This is not a mere performance optimisation, but it is often necessary to achieve a manageable size of the tables. In fact, the size of such tables is exponential in the induced width of the COP [2], which usually makes this approach not practical if all the rows are explicitly represented in the tables (e.g., assigning $-\infty$ to the unfeasible rows). In order to better understand our contribution to the GPU parallelisation of BE, in the following sections we provide a brief description of how composition and marginalisation are realised in Algorithm 1.

1) Composition: We now discuss how the $\oplus$ composition operator is implemented in Algorithm 1 by the join sum, an operation closely related to the inner join of relational algebra. For the remainder of this thesis, tables are represented according to Definition 2. Moreover, if $L$ is a tuple of elements, we refer to its $k^{\text {th }}$ element with $L[k]$. We adopt the zero-based numbering convention, i.e., tuples start at index 0 .

Definition 2: A table $T_{i}=\left\langle Q_{i}, d_{i}, R_{i}, \phi_{i}\right\rangle$ is defined by:

- $Q_{i} \subseteq X$, a tuple of variables called the scope of $T_{i}$;

- $d_{i}$, a tuple of natural numbers such that $d_{i}[k]=D_{j}$ is the size of the domain $Q_{i}[k]=x_{j}$, where $k \in\left\{1, \ldots,\left|Q_{i}\right|\right\}$;

- $R_{i}$, a tuple of rows: in particular, each row $R_{i}[k]$ is a tuple of natural numbers, defining a particular assignment of the variables in $Q_{i}$, where $k \in\left\{1, \ldots,\left|R_{i}\right|\right\}$;

- $\phi_{i}$, a tuple representing the actual values of the function, one for each row $R_{i}[k]$ : in particular, $\phi_{i}[k]$ is the value associated to the variable assignment represented by $R_{i}[k]$, where $k \in\left\{1, \ldots,\left|R_{i}\right|\right\}$.

\begin{tabular}{cccc|c}
\multicolumn{5}{c}{$T_{1}$} \\
\hline$x_{1}$ & $x_{3}$ & $x_{5}$ & $x_{8}$ & $\phi_{1}$ \\
\hline 0 & 1 & 0 & 1 & $\alpha_{0}$ \\
1 & 0 & 0 & 1 & $\alpha_{1}$ \\
1 & 1 & 0 & 1 & $\alpha_{2}$ \\
0 & 1 & 0 & 0 & $\alpha_{3}$ \\
0 & 0 & 0 & 1 & $\alpha_{4}$ \\
1 & 1 & 1 & 1 & $\alpha_{5}$ \\
\hline
\end{tabular}

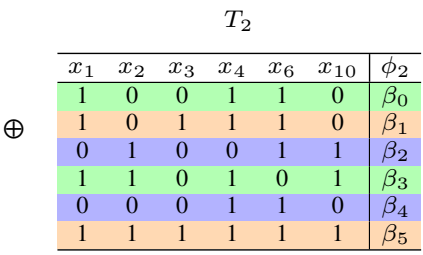

Fig. 1: Original tables $T_{1}$ and $T_{2}$.
Suppose we want to compute the join sum between $T_{1}$ and $T_{2}$ (shown in Figure 1), respectively associated to two tuples of variables $Q_{1}=\left\langle x_{1}, x_{3}, x_{5}, x_{8}\right\rangle$ and $Q_{2}=$ $\left\langle x_{1}, x_{2}, x_{3}, x_{4}, x_{6}, x_{10}\right\rangle$, with $Q_{1} \cap Q_{2}=\left\langle x_{1}, x_{3}\right\rangle$ representing the shared variables between $T_{1}$ and $T_{2}$. Notice that, as previously stated, some variable assignments are missing in $T_{1}$ and $T_{2}$, i.e., the unfeasible assignments.

A row in $T_{1}$ matches a row in $T_{2}$ if all the shared variables have the same values in both the rows (matching rows have been highlighted with the same colour in Figure 1). It is important to note that this is a many-to-many relationship, because multiple rows in the first table can match multiple rows in the second table. For instance

\begin{tabular}{cccc|c}
\hline$x_{1}$ & $x_{3}$ & $x_{5}$ & $x_{8}$ & $\phi_{1}$ \\
\hline 1 & 0 & 0 & 1 & $\alpha_{1}$ \\
\hline
\end{tabular} matches \begin{tabular}{ccccccc|c}
\cline { 3 - 8 } & $x_{1}$ & $x_{2}$ & $x_{3}$ & $x_{4}$ & $x_{6}$ & $x_{10}$ & $\phi_{2}$ \\
\hline 1 & 0 & 0 & 1 & 1 & 0 & $\beta_{0}$ \\
1 & 1 & 0 & 1 & 0 & 1 & $\beta_{3}$ \\
\hline
\end{tabular}

because they all have $x_{1}=1$ and $x_{3}=0$. Thus, the result table will have a row for each couple of matching rows in the input tables. In the above example, the corresponding rows in the result table $T_{1} \oplus T_{2}$ will be:

\begin{tabular}{cccccccc|c}
\hline$x_{1}$ & $x_{3}$ & $x_{5}$ & $x_{8}$ & $x_{2}$ & $x_{4}$ & $x_{6}$ & $x_{10}$ & $\phi_{\oplus}$ \\
\hline 1 & 0 & 0 & 1 & 0 & 1 & 1 & 0 & $\alpha_{1}+\beta_{0}$ \\
1 & 0 & 0 & 1 & 1 & 1 & 0 & 1 & $\alpha_{1}+\beta_{3}$ \\
\hline
\end{tabular}

These resulting rows are obtained combining the second row of $T_{1}$ and, respectively, the first and the fourth rows of $T_{2}$. They both have the same values for the shared variables $\left(x_{1}=1\right.$ and $\left.x_{3}=0\right)$. The values of the non-shared variables (i.e. $x_{5}$ and $x_{8}$ for $T_{1}$, and $x_{2}, x_{4}, x_{6}$ and $x_{10}$ for $T_{2}$ ) are copied from the corresponding matching rows. Hence, in the above example, $x_{5}=0$ and $x_{8}=1$ for both the resulting rows (since there is only one matching row in $T_{1}$ ), and $x_{2}=0$, $x_{4}=1, x_{6}=1$ and $x_{10}=0$ for the first resulting row (since it results from the match with the first matching row in $T_{2}$ ), and so on. Thus, the variable set of the resulting table is the union of the variable sets of the input tables. Finally, the values of the resulting rows are obtained summing the values of the corresponding matching rows, i.e. $\alpha_{1}+\beta_{0}$ and $\alpha_{1}+\beta_{3}$. Is it easy to see that if $n$ rows in $T_{1}$ match $m$ rows in $T_{2}$, they will result in $n \cdot m$ rows in the resulting table (Figure 2).

\begin{tabular}{cccccccc|c}
\hline$x_{1}$ & $x_{3}$ & $x_{5}$ & $x_{8}$ & $x_{2}$ & $x_{4}$ & $x_{6}$ & $x_{10}$ & $\phi_{\oplus}$ \\
\hline 0 & 0 & 0 & 1 & 1 & 0 & 1 & 1 & $\alpha_{4}+\beta_{2}$ \\
0 & 0 & 0 & 1 & 0 & 1 & 1 & 0 & $\alpha_{4}+\beta_{4}$ \\
1 & 0 & 0 & 1 & 0 & 1 & 1 & 0 & $\alpha_{1}+\beta_{0}$ \\
1 & 0 & 0 & 1 & 1 & 1 & 0 & 1 & $\alpha_{1}+\beta_{3}$ \\
1 & 1 & 0 & 1 & 0 & 1 & 1 & 0 & $\alpha_{2}+\beta_{1}$ \\
1 & 1 & 0 & 1 & 1 & 1 & 1 & 1 & $\alpha_{2}+\beta_{5}$ \\
1 & 1 & 1 & 1 & 0 & 1 & 1 & 0 & $\alpha_{5}+\beta_{1}$ \\
1 & 1 & 1 & 1 & 1 & 1 & 1 & 1 & $\alpha_{5}+\beta_{5}$ \\
\hline
\end{tabular}

Fig. 2: Join sum result $T_{\oplus}$.

2) Marginalisation: The second fundamental operation, which implements the $\Downarrow$ marginalisation operator in Algorithm 1 , is the maximisation. Suppose that, as a result of the inner join sum operation at line 13 of Algorithm 1, we obtain the table $T$ in Figure 3.

Now, suppose that $x_{p}=x_{8}$. Then, Algorithm 1 requires to maximise such table marginalising out $x_{8}$, i.e., removing the column corresponding to $x_{8}$. As a result of this removal, some 


\begin{tabular}{cccc|c}
\hline$x_{1}$ & $x_{3}$ & $x_{5}$ & $x_{8}$ & $\phi$ \\
\hline 0 & 0 & 0 & 0 & $\alpha_{0}$ \\
0 & 0 & 0 & 1 & $\alpha_{1}$ \\
1 & 0 & 0 & 0 & $\alpha_{2}$ \\
1 & 0 & 0 & 1 & $\alpha_{3}$ \\
1 & 1 & 0 & 1 & $\alpha_{4}$ \\
1 & 1 & 1 & 1 & $\alpha_{5}$
\end{tabular}

Fig. 3: Initial table $T$.

\begin{tabular}{ccc|c}
\hline$x_{1}$ & $x_{3}$ & $x_{5}$ & $\phi_{\Downarrow}$ \\
\hline 0 & 0 & 0 & $\max \left(\alpha_{0}, \alpha_{1}\right)$ \\
1 & 0 & 0 & $\max \left(\alpha_{2}, \alpha_{3}\right)$ \\
1 & 1 & 0 & $\alpha_{4}$ \\
1 & 1 & 1 & $\alpha_{5}$ \\
\hline
\end{tabular}

Fig. 4: Maximisation result. rows may now be equal considering the remaining columns (e.g., $R[1]$ and $R[2]$ both contain $\langle 0,0,0\rangle$ in the first three columns, as well as $R[3]$ and $R[4]$, which contain $\langle 1,0,0\rangle$ ). Since one cannot have duplicate rows, the maximisation operations computes a single row that, as a value, stores the maximum of the original values. ${ }^{2}$ The final result of the maximisation of $T$ can be seen in Figure 4 .

Composition and marginalisation operators are also employed by more modern versions of BE, e.g., Bucket-Tree Elimination (BTE) proposed by Kask, Dechter, Larrosa, et al. [20], hence our contributions are valuable also in the context of these newer algorithms. Here we focus on BE since it was the first version of these message-passing techniques to tackle constrained optimisation, and its performance is generally comparable with BTE, which, in turn, is optimised for some specific problems, i.e., singleton-optimality problems.

Notice that DP (and in particular BE) is not the only approach to solve COPs, which can also be tackled with DFS-based approaches [21]. While they represent important techniques in the context of constrained optimisation, we do not focus on these algorithms in the context of this paper, since DFS is known to be difficult to parallelise [22].

We now discuss BP on JTs, which is a close variation of $\mathrm{BE}$ [2] that is also based on composition and marginalisation operators (i.e., scattering and reduction respectively).

3) Belief Propagation on Junction Trees: Belief Propagation on Junction Trees [6] (BP on JTs) is used to propagate inference on a Bayesian Network (BN), a representation of a joint distribution over a set of $n$ random variables $X$, structured as a directed acyclic graph whose vertices are the random variables and the directed edges represent dependency relationships among the random variables. The propagation of beliefs (or posteriors) runs over a Junction Tree (JT), generated from a $\mathrm{BN}$ by means of moralisation and triangulation [6]. Every vertex $N_{i}$ of the JT contains a set $Q_{i} \subseteq X$ of random variables that forms a maximal clique in the moralised and triangulated $\mathrm{BN}$, each associated to a potential table represented by $T_{i}=\left\langle Q_{i}, d_{i}, R_{i}, \phi_{i}\right\rangle$ according to Definition 2. In standard $\mathrm{BP}, R_{i}$ contains all the possible variable assignments over their domains, hence $\left|R_{i}\right|=\left|\phi_{i}\right|=\prod_{k=1}^{\left|Q_{i}\right|} d_{i}[k]$. Assuming that $T_{i}$ and $T_{j}$ are potential tables associated to adjacent vertices in the JT, we associate a separator table $S e p_{i j}=\left\langle Q_{i j}, d_{i j}, R_{i j}, \phi_{i j}\right\rangle$ to the edge $\left(N_{i}, N_{j}\right)$, whose scope $Q_{i j}$ is represented by the shared variables between the two tables, i.e., $Q_{i j}=Q_{i} \cap Q_{j}$.

BP on JTs is then invoked whenever we receive new evidence for a particular set of variables $\mathcal{E} \subseteq X$, updating the potential tables associated to the $\mathrm{BN}$ in order to reflect this new information. To this end, a two-phase procedure is employed:

\footnotetext{
${ }^{2}$ If we marginalise out $x_{i}$, we maximise over up to $d_{i}$ rows
}

first, in the evidence collection phase, messages are collected from each vertex $N_{i}$, starting from the leaves all the way up to a designated root vertex. Then, during evidence distribution, messages are sent from the root to the leaves. In both phases, each recursive call comprises a MESSAGEPASS procedure, which implements the propagation of beliefs between the potential tables $T_{i}$ and $T_{j}$ in two steps:

1) Reduction: the potential table $S e p_{i j}$ is updated to $S e p_{i j}^{*}$. In particular, each row of $S e p_{i j}^{*}$ is obtained summing the corresponding rows of $T_{i}$, i.e., the ones with a matching variable assignment. Reduction implements the $\Downarrow$ marginalisation operator of $\mathrm{BE}$, which is achieved with a summation in this case.

2) Scattering: $T_{j}$ is updated with the new values of $S e p_{i j}^{*}$, i.e., every row of $T_{j}$ is multiplied for the ratio between the corresponding rows in $S e p_{i j}^{*}$ and $S e p_{i j}$. Following Zheng and Mengshoel [18], we assume that $\frac{0}{0}=0$. Scattering implements the composition with the product operation, and it corresponds to the $\oplus$ operator of $\mathrm{BE}$.

In both BE and BP on JTs, it is easy to see that the message passing phase require several independent computations spanning over multiple rows of the tables, suggesting a multithreaded algorithm in which such degree of parallelism can be exploited by means of GPUs.

\section{B. GPU Architecture}

GPUs are designed for compute-intensive, highly parallel computations. To this end, more transistors are devoted to data processing rather than data caching and flow control. These architectures are especially well-suited for problems that can be expressed as data-parallel computations where data elements are mapped to parallel processing threads. The GPU (device) is mainly employed to implement compute-intensive parts of an application, while control-dominant computations are performed by the CPU (host). In our approach, the GPU is programmed using the CUDA programming framework [10], which requires the definition of a kernel, a particular routine executed in parallel by thousands of threads on different inputs. Threads are organised in thread blocks, sharing fast forms of storage and synchronisation primitives. On the other hand, physical and design constraints limit the number of threads per block, since all the threads of a block are expected to reside on the same Streaming Multiprocessor (SM) and must share limited memory resources. Memory management is a crucial aspect in the design of efficient GPU algorithms, since memory accesses are particularly expensive and have a significant impact on performance. As a consequence, memory accesses must be carefully devised to achieve high computational throughput (see Section V-A). In what follows, we discuss previous approaches for BE-related algorithms that can be found in the parallel computing literature.

\section{Related Work}

BP on JTs represents a well-known inference algorithm, which has received significant attention in the parallel computing literature due to its high computational demands. In 
particular, Xia and Prasanna [23] propose a distributed approach that combat this by decomposing the initial JT into a set of subtrees, and then they perform the evidence propagation in the subtrees in parallel on a cluster. In this paper we also focus on exploiting parallel architectures for BP on JTs, but, in contrast, we aim at parallelising the single propagation operation, which is the most computationally intensive task of the entire algorithm.

The most recent work addressing the parallelisation of BP on GPUs is presented by Zheng, Mengshoel, and Chong [17]. In particular, the authors pursue the same goal tackled by our approach discussed in Section III, i.e., parallelise the atomic operations of propagation so that it could be embedded in different algorithms. On the other hand, such an approach proposes a different parallelisation strategy, devising a twodimensional parallelism, in which an higher level elementwise parallelism is stacked on top of a lower level arithmetic parallelism, to better exploit the massive computational power provided by modern GPUs. In particular, elementwise parallelism is achieved by computing each of the $\left|R_{i j}\right|$ reduction-and-scattering operations in parallel, which require $\left|R_{i j}\right|$ mapping tables (one per row of $S e p_{i j}$ ) to allow each concurrent task to correctly locate its input data from the corresponding potential tables. On the other hand, arithmetic parallelism represents the multi-threaded computation of each reduction-and-scattering operation, by means of well known parallel algorithms that can be found in literature [10].

Although this approach represents a significant contribution to the state-of-the-art, there are some drawbacks that hinder its applicability. In particular, the proposed memory layout is not optimised for GPUs, for three main reasons:

- Threads need to access data in sparse and discontinuous memory locations using an additional indexing table, breaking coalescence and drastically reducing the throughput of memory transfers (Figure 8). Coalescence is crucial and it should be exploited in order to reduce memory accesses to the global memory, improving the compute-to-memory ratio and achieving a greater computational throughput.

- Since input data is organised in a discontinuous pattern rather than in continuous chunks, it is mandatory to transfer the entire potential tables to the global memory of the GPU before starting the computation of the BP algorithm, hindering two desirable properties: i) this approach is not applicable to potential tables that do not fit into global memory, since the sparsity of the data prevents any possibility of splitting them into smaller parts, and ii) since the computation cannot be started before the entire input data has been copied to the GPU, the cost of memory transfers cannot be amortised by means of technologies like NVIDIA CUDA streams.

Moreover, the authors devise this technique for BP, where tables are complete (i.e., they include a row for every possible assignment of the variables in their scope). Thus, this approach cannot be applied to problems in which tables are incomplete, e.g., COPs and WCSPs.

In the context of COPs, the only work that specifically focuses on the implementation of the $\mathrm{BE}$ algorithm for many- cores architectures is the one by Fioretto, Le, Pontelli, et al. [24], in which the authors devise an algorithm to realise the join sum and the maximisation operations (referred as aggregate and project) on GPUs, by exploiting the high degree of parallelism inherent in these operations. Although this approach represents a significant contribution to the state-ofthe-art, there are some drawbacks that hinder its applicability. First, the indexing of the tables is executed by using a Minimal Perfect Hash function, i.e., a hash function that maps $n$ keys to $n$ consecutive integers, which can be easily adopted as the indices of such keys. Although minimal perfect hash functions can be used in parallel by different threads to index the input, their construction is inherently sequential, since the index of a key depends on the indices assigned to the previously considered keys [25]. This aspect reduces the efficiency of this approach especially on big instances, as shown by our experiments in Section VI-B.

To overcome these limitations, we propose a better way to tackle the GPU computation of the message-passing phase of BE. In particular, we first present a technique that improves the parallelisation of BP with complete tables (already published in [26]), and then we extend it in order to be applicable to the more general case of incomplete tables.

\section{Processing Complete Tables}

In this section, we detail our contribution to the GPU computation of messages in BP, exploiting the fact that, in such problem, tables are complete. In particular, we first discuss how we preprocess potential tables in order to index their rows efficiently and achieve coalesced memory accesses. Then, this table layout is exploited by the actual CUDA kernel, which executes the actual message passing phase of BP through highly efficient routines.

\section{A. Table Preprocessing}

Suppose we have to propagate new evidence from the potential table $T_{1}$ to the potential table $T_{2}$, respectively associated to two tuples of variables $Q_{1}=\left\langle x_{3}, x_{2}, x_{1}\right\rangle$ and $Q_{2}=\left\langle x_{5}, x_{4}, x_{1}\right\rangle$, with the shared variables $Q_{12}=Q_{1} \cap Q_{2}=\left\langle x_{1}\right\rangle$. We assume that $x_{1}, x_{3}$ and $x_{5}$ are binary variables, while $x_{2}$ and $x_{4}$ can assume 3 values. In the approach by Zheng and Mengshoel [18], each row of the separator table $S e p_{12}$ is assigned to a different block of threads, which are responsible of the reduction of the rows of $T_{1}$ with a matching variable assignment and the subsequent scattering on matching rows in $T_{2}$. In Figure 5, rows associated to different blocks of threads have been marked in different colours, i.e., white and grey for $x_{1}=0$ and $x_{1}=1$ respectively. The organisation of input data provided by these tables is undesirable for GPU architectures. In fact, threads responsible of the computation of white rows cannot access consecutive memory addresses, as their data is interleaved with grey rows, thus breaking memory coalescence. Moreover, even if the computation of white rows requires half of the input data, its sparsity forces us to transfer the entire tables to the global memory before starting the algorithm. We propose to solve these issues by means of a preprocessing phase, in which rows associated to the same row in $S_{e p} p_{12}$ (i.e., rows of the 

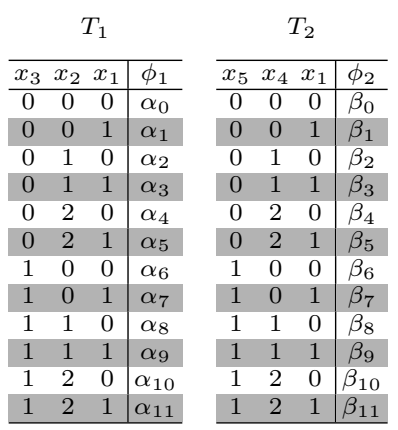

Fig. 5: Original tables.

\begin{tabular}{|c|c|c|c|c|c|c|c|}
\hline \multicolumn{4}{|c|}{$T_{1}^{p}$} & \multicolumn{4}{|c|}{$T_{2}^{p}$} \\
\hline$x_{1}$ & $x_{3}$ & $x_{2}$ & $p\left(\phi_{1}\right)$ & $\overline{x_{1}}$ & $x_{5}$ & $x_{4}$ & $p\left(\phi_{2}\right)$ \\
\hline$\overline{0}$ & 0 & 0 & $\alpha_{0}$ & 0 & 0 & 0 & $\beta_{0}$ \\
\hline 0 & 0 & 1 & $\alpha_{2}$ & 0 & 0 & 1 & $\beta_{2}$ \\
\hline 0 & 0 & 2 & $\alpha_{4}$ & 0 & 0 & 2 & $\beta_{4}$ \\
\hline 0 & 1 & 0 & $\alpha_{6}$ & 0 & 1 & 0 & $\beta_{6}$ \\
\hline 0 & 1 & 1 & $\alpha_{8}$ & 0 & 1 & 1 & $\beta_{8}$ \\
\hline 0 & 1 & 2 & $\alpha_{10}$ & 0 & 1 & 2 & $\beta_{10}$ \\
\hline 1 & 0 & 0 & $\alpha_{1}$ & 1 & 0 & 0 & $\beta_{1}$ \\
\hline 1 & 0 & 1 & $\alpha_{3}$ & 1 & 0 & 1 & $\beta_{3}$ \\
\hline 1 & 0 & 2 & $\alpha_{5}$ & 1 & 0 & 2 & $\beta_{5}$ \\
\hline 1 & 1 & 0 & $\alpha_{7}$ & 1 & 1 & 0 & $\beta_{7}$ \\
\hline 1 & 1 & 1 & $\alpha_{9}$ & 1 & 1 & 1 & $\beta_{9}$ \\
\hline 1 & 1 & 2 & $\alpha_{11}$ & 1 & 1 & 2 & $\beta_{11}$ \\
\hline
\end{tabular}

Fig. 6: Preprocessed tables.

same colour, in the above example) are stored in consecutive addresses in the corresponding potential tables, as shown in Figure 6. Threads responsible of white rows execute coalesced memory accesses, and start the computation while grey rows are still being transferred to the GPU. Each block of threads easily retrieves its input data without any costly mapping table, in contrast with the approach by Zheng and Mengshoel [18].

Consider $T_{1}^{p}=\left\langle Q_{1}^{p}, d_{1}^{p}, R_{1}^{p}, \phi_{1}^{p}\right\rangle$ in Figure 6, resulting from a permutation $\sigma$ of $Q_{1}$ in which the variables in $Q_{12}$ are brought to the Most Significant ${ }^{3}$ (MS) positions in $Q^{p}=\sigma(Q)$. In this way, we can assure that rows with the same assignment of the variables in $Q_{12}$ form a contiguous chunk of memory.

Our table representation by means of ordered tuples imposes that $d^{p}, R^{p}$ and $\phi^{p}$ are coherently defined, to guarantee the equivalence to the original table. While the former can be easily obtained by applying $\sigma$ to $d$, the computation of $R^{p}$ can be avoided, therefore only $\phi^{p}$ requires a particular dissertation, which is covered in the following sections.

1) Table Indexing: Since in any table $T=\langle Q, d, R, \phi\rangle, R$ contains all the possible variable assignments, we can avoid storing $R$ in memory. In fact, since the order of variables is fixed, given any row $r=R[k], k$ can be computed with:

$$
k=\sum_{i=1}^{|Q|-1}(r[i] \underbrace{\prod_{j=i+1}^{|Q|} d[j]}_{\mathcal{D}[i]})+r[|Q|]=\sum_{i=1}^{|Q|-1}(r[i] \cdot \mathcal{D}[i])+r[|Q|]
$$

where $r[i]$ represents the value assumed by the variable $Q[i]$ in $r$. Each $\mathcal{D}[i]$ represents the product of all the elements starting from position $i+1$ in $d$, hence we refer to the tuple $\mathcal{D}$ as the exclusive postfix product of $d$. Such an operation can be seen as a variation of the standard exclusive prefix sum operation, in which the result is computed by summing all the elements up to $i-1$. We define $\mathcal{D}[|Q|]:=1$ (the identity element for the product), similarly to the definition of the first element of the exclusive prefix sum as 0 .

On the other hand, each $r[i]$ can be retrieved from $k$ as $r[i]=\lfloor k / \mathcal{D}[i]\rfloor \bmod d[i]$. Thus, $R$ can be dropped from our representation in memory, hence, as previously claimed, the computation of $R^{p}$ is unnecessary. For a better understanding, let $r$ with $Q=\left\langle x_{1}, x_{2}, x_{3}\right\rangle$, and $d=\langle 2,16,10\rangle$ :

$$
r=\begin{array}{ccc|c}
\hline x_{1} & x_{2} & x_{3} & \phi \\
\hline 1 & 10 & 7 & v_{267} \\
\hline
\end{array}
$$

\footnotetext{
${ }^{3}$ Variables are listed from the most significant to the least significant.
}

From Equation $1, r$ is in position $k=1 \cdot d[2] \cdot d[3]+10 \cdot d[3]+$ $7=267$ in $\phi$. Moreover, $x_{1}=1=\lfloor 267 / \mathcal{D}[1]\rfloor \bmod d[1], x_{2}=$ $10=\lfloor 267 / \mathcal{D}[2]\rfloor \bmod d[2]$ and $x_{3}=7=\lfloor 267 / \mathcal{D}[3]\rfloor \bmod d[3]$.

As mentioned before, to maintain a coherent representation of the preprocessed table $T^{p}=\left\langle Q^{p}, d^{p}, R^{p}, \phi^{p}\right\rangle$, the values in $\phi$ must be correctly permuted into $\phi^{p}$.

2) Table Reordering: This section will cover our approach to achieve the column reordering detailed in Section III-A. As mentioned before, we do not store $R$, since each row $r \in R$ can be retrieved from its index with the above detailed technique, hence the computation of $R^{p}$ will not be covered. On the other hand, for any $\phi[k]$ at index $k$ in $\phi$ it is necessary to compute its index $k^{p}$ in the preprocessed table $T^{p}$ to compute $\phi^{p}$.

A naive approach would require to apply the permutation $\sigma$ on each row $r=R[k]$, which comprises 3 steps: for each $k$, compute the corresponding variable assignment $\langle r[1], \ldots, r[i], \ldots, r[|Q|]\rangle$, apply $\sigma$ on the now available sequence of $r[i]$ and, finally, obtain $k^{p}$ using Equation 1. Since each of the 3 above mentioned steps has a complexity of $O(|Q|)$, such approach requires $O(3|\phi||Q|)$.

In what follows, we show a more efficient approach to calculate $k^{p}$. For simplicity, we first explain how to compute the index resulting from swapping the variables at positions $i$ and $j$. Then, we provide an algorithm to compute $k^{p}$ by means of a sequence of swaps.

Proposition 1: Given $T=\langle Q, d, R, \phi\rangle$ and $T^{s}=\left\langle Q^{s}, d^{s}\right.$, $\left.R^{s}, \phi^{s}\right\rangle$, where $Q^{s}$ and $d^{s}$ has been respectively obtained swapping $Q[i]$ with $Q[j]$ and $d[i]$ with $d[j]$ (with $i>j$ ), $\phi^{s}$ is a permutation of $\phi$, i.e., $\phi[k]=\phi^{s}\left[k^{\prime}\right]$ and $k^{\prime}$ is:

$$
\begin{aligned}
k^{\prime} & =r[1] \cdot d[2] \cdots d[i] \cdots d[j] \cdots d[|Q|]+\cdots \\
& +r[i] \cdot d[j+1] \cdots d[j] \cdots d[|Q|] \\
& +r[j+1] \cdot d[j+2] \cdots d[|Q|]+\cdots+r[i-1] \cdot d[j] \cdots d[|Q|] \\
& +r[j] \cdot d[i+1] \cdots d[|Q|] \\
& +r[i+1] \cdot d[i+2] \cdots d[|Q|]+\cdots+r[|Q|]
\end{aligned}
$$

Then, $k^{\prime}=f(k, i, j)$ can also be calculated as:

$$
\begin{aligned}
& k^{\prime}=\frac{(2 a)}{k-k \bmod \mathcal{D}[j-1]}+\overleftarrow{\mathcal{D}[j] \cdot d[j] / d[i] \cdot\lfloor k / \mathcal{D}[i]] \bmod d[i]}+\frac{(2 e)}{k \bmod \mathcal{D}[i]} \\
& \left(2 c^{\prime}\right) \\
& +\underbrace{d[j] / d[i] \cdot \cdot(k \bmod \mathcal{D}[j]-k \bmod \mathcal{D}[i-1])}_{(2 c)}+\underbrace{\left.\mathcal{D}[i] \cdot{ }^{k} / \mathcal{D}[j]\right] \bmod d[j]}_{(2 d)}
\end{aligned}
$$

Proof: The proof is in the supplementary materials.

Proposition 1 is then used to reorder any potential table $T$ according to the layout detailed in Section III-A. More formally, let $\mathcal{S}=\left\langle\mathcal{S}_{1}, \ldots, \mathcal{S}_{n}\right\rangle$ be a sequence of $n$ swaps, each represented by an ordered ${ }^{4}$ pair of positions $\mathcal{S}[i]=\left\langle a_{i}, b_{i}\right\rangle$, so that we permute $Q$ into $\sigma(Q)$ (moving the desired subset of variables to the MS positions) by means of the sequence of $i$ swaps of the variables in positions $a_{i}$ and $b_{i}$, as described in Proposition 1. Then, $\phi^{p}$ is computed with Algorithm 2.

The tuple of swaps $\mathcal{S}$ required to move $\left|Q_{12}\right|$ variables to the MS positions of the tables $T_{1}$ and $T_{2}$ is computed as follows. Consider $T_{1}$ and let us assume that $s$ shared variables (with $\left.0 \leq s \leq\left|Q_{12}\right|\right)$ are already within the first $\left|Q_{12}\right|$ positions of the corresponding variable tuple $Q_{1}$. Then, it is sufficient to swap

\footnotetext{
${ }^{4}$ We assume that, for every pair $\left\langle a_{i}, b_{i}\right\rangle, a_{i}>b_{i}$.
} 


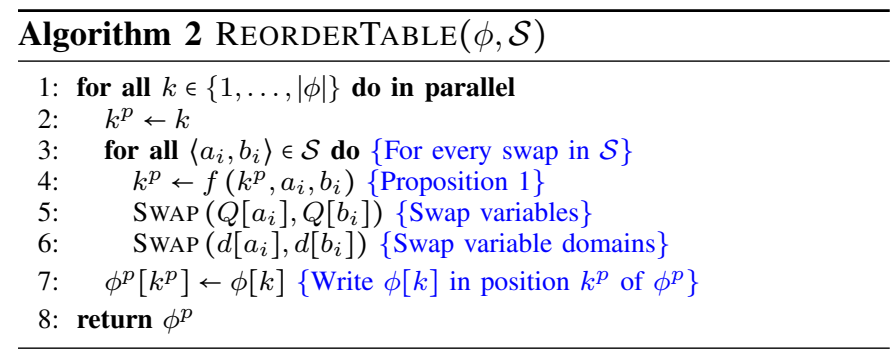

the $\left|Q_{12}\right|-s$ shared variables with index greater than $\left|Q_{12}\right|$ with the non-shared ones which are placed within the first $\left|Q_{12}\right|$ positions. On the other hand, table $T_{2}$ can be preprocessed by swapping each shared variable $Q_{2}[h]$ with $Q_{2}[k]$ such that $Q_{2}[h]=Q_{1}[k]$ for $k \in\left\{1, \ldots,\left|Q_{12}\right|\right\}$. This algorithm ensures the same order of the variables in $Q_{12}$ in both tables.

As an example, we reorder the row $R_{1}[10]=\langle 1,2,0\rangle$ in position $k=10$ of $T_{1}$ in Figure 5 and compute its index $k^{p}$ in $T_{1}^{p}$. In this case, the desired order is obtained with $\mathcal{S}[1]=$ $\langle 3,1\rangle$ and $\mathcal{S}[2]=\langle 3,2\rangle^{5}$, i.e, by swapping $Q[3]=x_{1}$ with $Q[1]=x_{3}$, then swapping $Q[3]=x_{3}$ with $Q[2]=x_{2}$. Initially, $d[3]=d[1]=2, \mathcal{D}[3]=1$ and $\mathcal{D}[1]=6$. Then, applying Proposition 1 to the row with index $k=10$ results in $(2 a)=$ $(2 e)=0$ (since there are no variables before $x_{3}$ and after $x_{1}$ ), $(2 b)=6 \cdot 2 / 2 \cdot 0=0,(2 c)=2 / 2(10 \bmod 6-10 \bmod 2)=4$ and $(2 d)=1 \cdot 1=1$, hence $f(10,3,1)=5$, meaning that $\alpha_{10}$ would have index 5 after $\mathcal{S}[1]$. To compute its final index, we apply $\mathcal{S}[2]=\langle 3,2\rangle$. At this point $Q_{1}=\left\langle x_{1}, x_{2}, x_{3}\right\rangle, \mathcal{D}[3]=1$, $\mathcal{D}[2]=d[3]=2$ and $d[2]=3$, hence $(2 c)=(2 e)=0$ (since there are no variables after $x_{3}$ and between $x_{2}$ and $\left.x_{3}\right)$. On the other hand, $(2 a)=5-5 \bmod 6=0,(2 b)=2 \cdot 3 / 2 \cdot 1=3$ and $(2 d)=1 \cdot 2=2$, thus $\phi^{p}[5]=\alpha_{10}\left(\right.$ see $\left.T_{1}^{p}\right)$.

Algorithm 2 provides a method to rearrange any couple of potential tables $T_{i}$ and $T_{j}$ such that the variables of their separator are moved to the MS positions (see Section III-A). We now analyse the impact of this preprocessing phase on the overall performance of the algorithm, by showing how it is more efficient than the naive approach mentioned above.

3) Computational Complexity:

Proposition 2: Algorithm 2 has a time complexity of $O(|\phi||\mathcal{S}|) \leq O\left(|\phi|\left|Q_{12}\right| / 2\right)<O(|\phi||Q|)$.

Proof: The proof is in the supplementary materials.

In our experimental evaluation, we performed the variable ordering with an average of $|\mathcal{S}|=3$ swaps, resulting in an improvement of an order of magnitude w.r.t. the naive approach, which, in contrast, requires tens of operations for each row. It is important to note that this preprocessing phase is done once for all, while compiling the BN in the corresponding JT. In fact, the acquisition of new evidence does not change the structure of the network itself, hence we can avoid to reorder each potential table at each BP by storing and updating the couple of corresponding reordered tables for each separator. Even if Algorithm 2 does not reorder $\phi$ in-place, the additional space required to store $\phi^{p}$ is amortised by discarding the original table, since it is not needed in any subsequent phase of the algorithm. Furthermore, each iteration of the external

\footnotetext{
${ }^{5}$ Swapping $x_{3}$ and $x_{2}$ is not necessary since neither of them belongs to $Q_{12}$, but it has been included in our example to better explain the algorithm.
}

loop (Lines 1-7) of Algorithm 2 is independent and can be computed in parallel. As a consequence, the worst-case time complexity of the parallel version of Algorithm 2 is $O(|\phi||\mathcal{S}| / t)$, where $t$ is the number of threads. Given a $\mathbf{J T}=(V, E)$, our algorithm needs to store a couple of potential tables for each separator. Since threads can index input rows on-the-fly, mapping tables can be avoided. Thus, the memory requirements are $O(2 \cdot|E|)$. In contrast, the approach proposed by Zheng and Mengshoel [18] maintains one potential table for each clique, but it needs two mapping tables for each separator table. Hence, it requires $O(V+2 \cdot|E|)$ tables.

\section{B. GPU Kernel Implementation}

In our approach to BP on GPUs, each block of threads is responsible for one element of the separator table, which is associated to a corresponding group of rows in potential tables. Such high-level organisation of the computation allows us to carry out the entire reduction and scattering stages within a single thread block, hence avoiding any costly inter-block synchronisation structure. On one hand, the performance of our algorithm clearly benefits from the lack of interdependence among different blocks, which would reduce the overall computation parallelism. On the other hand, since the size of thread blocks has an intrinsic limit imposed by the hardware architecture (e.g., 2048 threads in Kepler GPUs), the proposed organisation may serialise part of the workload if the number of rows to manage exceeds such limit. Nevertheless, such an issue is not problematic in our test cases, since the above mentioned case rarely verifies. In fact, in our experimental evaluation, each block has to reduce an average of 14 elements, ${ }^{6}$ hence allowing a full parallelisation.

If the serialisation is small (i.e., each thread has to reduce and scatter few rows), the effect on the overall performance is negligible. This is due to the fact that the task is computed extremely efficiently in thread-private memory space using registers. In what follows, we explain the actual implementation of the above mentioned concepts in detail.

1) Reduction: Once the input data is in the shared memory, the kernel starts the reduction phase that, in our approach, is implemented with the NVIDIA CUB library ${ }^{7}$ by means of a block reduce raking algorithm. The algorithm consists of three steps: i) an initial sequential reduction in registers (if each thread contributes to more than one input), in which warps other than the first one place their partial reductions into shared memory, ii) a second sequential reduction in shared memory, in which threads within the first warp accumulate data by ranking across segments of shared partial reductions, and iii) a final reduction within the raking warp based on the KoggeStone algorithm [27] produces the final output. This scheme is particularly efficient, since it involves a single synchronisation barrier after the first phase it incurs zero bank conflicts ${ }^{8}$ for primitive data types. On newer CUDA architectures (e.g.,

\footnotetext{
${ }^{6}$ This is the average, over all BNs, of the ratio between the average potential table size and the average separator table size (see Table I).

${ }^{7}$ Available at http://nvlabs.github.io/cub.

${ }^{8}$ If multiple memory accesses map to the same memory bank, the accesses are serialised and split into as many separate conflict-free requests as necessary, thus decreasing the effective bandwidth.
} 
NVIDIA Kepler), such implementation exploits shuffle instructions, which are a new set of primitives provided by the CUDA programming language. Shuffle instructions enable threads within the same warp to exchange data through direct register accesses, hence avoiding shared memory accesses and improving the computational throughput of the algorithm.

In particular, such scheme is collectively performed by the block of threads associated to a particular element of the separator table, in order to compute its updated value as the sum of the corresponding rows of the first potential tables, i.e., the ones with a matching variable assignment. Once the reduction of the entire chunk has been completed, the first thread computes the value of $R_{i j}$ assigned to the considered block, which serves as input for the subsequent scattering phase of belief propagation.

2) Scattering: The final stage of BP consists of the scattering operation, which performs the actual update of $T_{2}^{p}$ by means of $R_{i j}$ computed in the above mentioned phase. The implementation of such operation benefits from the proposed memory layout, since it is realised with maximum parallelism and computational throughput. Each row of $T_{2}^{p}$ is assigned to one thread, which multiplies its current value for $R_{i j}$, computed in the reduction phase. Once the kernel has been executed by all blocks, the propagation of belief has completed the inclusion of new evidence in $T_{2}^{p}$, which can be finally transferred back to the CPU memory.

Notice that our method of computing the message passing phase of BP does not affect the semantic of the algorithm, hence the results computed by our techniques are correct.

It is important to note that all the techniques described in the current section are based on the fact that, in BP on JTs, tables are complete, i.e., they contain all the possible variable assignments. Therefore, this approach cannot be directly applied to solve COPs with $\mathrm{BE}$, where, as explained in Section II-A, constraints make some variable assignments unfeasible and, hence, tables are incomplete. To overcome this issue, we propose a generalised approach able to process incomplete tables, as explained in the following section.

\section{Processing Incomplete TABles}

In this section we elaborate our contribution to the parallelisation of the BE algorithm to solve COPs. We propose a novel method to implement the join sum and the maximisation operations (Line 13 of Algorithm 1) on GPUs, to tackle their computational complexity and speedup the execution of BE.

\section{A. Table Preprocessing}

In order to explain our approach, we contextualise it on the example introduced in Section II-A1 (Figure 1). The goal is to rearrange the rows of these tables so to have the same final placement discussed in Section III-A, since the current data organisation suffers from very poor data locality.

In particular, the preprocessing of these tables aims at achieving two fundamental requirements: i) rows of the same colour should be in consecutive memory addresses, to have full coalescence in memory accesses and to reduce the sparsity of data; ii) coloured groups should be in the same order (considering the set of shared variables) in both tables, to locate them efficiently when computing the join sum result. This is required since tables can be incomplete. We achieve these objectives by means of Algorithm 3. The first step of the preprocessing phase requires to move the $\left|Q_{12}\right|$ columns corresponding to the shared variables to the $\left|Q_{12}\right|$ Least Significant ${ }^{3}$ (LS) places. Notice that this step is an embarrassingly parallel [10] task, and it can be trivially divided among $|R|$ threads, each independently processing a single row. Subsequently, the algorithm reorders $R$ and $\phi$ by means of a LSD radix sort algorithm [15] (implemented with the NVIDIA CUB library ${ }^{7}$ ). It is not necessary to adopt a radix sort algorithm in this phase (as every sorting algorithm that operates on the basis of the LS $\left|Q_{12}\right|$ places would work). However, we decide to use such an algorithm since it can be parallelised very efficiently [15]. As a final step, the algorithm remove the non-matching groups of rows (white rows in Figure 1), since they do not generate any output row in the result table, obtaining the preprocessed tables $T_{1}^{p}$ and $T_{2}^{p}$ in Figure 7 . Notice that none of these three steps requires to have an entire table stored in the global memory, thus it is possible to easily split the input tables into manageable chunks meeting the memory capabilities of the GPU and preprocess them. ${ }^{9}$

\begin{tabular}{cccc|c}
\multicolumn{7}{c}{$T_{1}^{p}$} \\
\hline$x_{5}$ & $x_{8}$ & $x_{1}$ & $x_{3}$ & $\phi_{1}^{p}$ \\
\hline 0 & 1 & 0 & 0 & $\alpha_{4}$ \\
0 & 1 & 1 & 0 & $\alpha_{1}$ \\
0 & 1 & 1 & 1 & $\alpha_{2}$ \\
1 & 1 & 1 & 1 & $\alpha_{5}$ \\
\hline
\end{tabular}

\begin{tabular}{ccccccc|c}
\multicolumn{10}{c}{$T_{2}^{p}$} \\
\cline { 2 - 7 } & \multicolumn{1}{c}{$x_{6}$} & $x_{10}$ & $x_{2}$ & $x_{4}$ & $x_{1}$ & $x_{3}$ & $\phi_{2}^{p}$ \\
\hline 1 & 1 & 1 & 0 & 0 & 0 & $\beta_{2}$ \\
1 & 0 & 0 & 1 & 0 & 0 & $\beta_{4}$ \\
1 & 0 & 0 & 1 & 1 & 0 & $\beta_{0}$ \\
0 & 1 & 1 & 1 & 1 & 0 & $\beta_{3}$ \\
1 & 0 & 0 & 1 & 1 & 1 & $\beta_{1}$ \\
1 & 1 & 1 & 1 & 1 & 1 & $\beta_{5}$ \\
\hline
\end{tabular}

Fig. 7: Final preprocessed tables.

We now discuss how it is possible to exploit this row layout to index these tables and have multiple thread efficiently locate their input to compute the join sum in parallel on the GPU.

\section{B. Join Sum GPU Computation}

Join sum implements the composition operation of BE. Our method adopts a gather paradigm [10], in which each thread is responsible for the computation of exactly one element of the output. Such a paradigm offers many advantages w.r.t. the counterpart approach, i.e., the scatter paradigm, in which each thread is associated to one element of input and contributes to the computation of many output elements. Scatter-based approaches have a reduced independence of the operations and they require atomic primitives (which serialise parts of the

\footnotetext{
${ }^{9}$ If it is necessary to sort a table larger that the GPU global memory, it is possible to split it into chunks, sort each of them (using the above mentioned radix sort algorithm), and then merge the sorted chunks (adopting the merge sort algorithm) on the CPU.
}

\begin{tabular}{l}
\hline Algorithm 3 PREPROCESSINCOMPLETE $\left(T_{1}, T_{2}\right)$ \\
\hline 1: Move shared variables in $Q_{1}$ and $Q_{2}$ to the $\left|Q_{12}\right|$ LS places \\
2: Sort $R_{1}, R_{2}, \phi_{1}, \phi_{2}$ using a LSD radix sort on the $\left|Q_{12}\right|$ LS places \\
3: Remove row groups that do not match between $T_{1}$ and $T_{2}$ \\
\hline
\end{tabular}


computation) to avoid race conditions, when multiple input elements concurrently modify the same output element.

In our particular case, one thread computes one particular output row at index $i$ (i.e., both $R[i]$, the variable assignment part, and $\phi[i]$, the value part), on the basis of the two input rows associated, which can be identified as explained below. First, we compute the number of rows in each coloured group for $T_{1}$ and $T_{2}$. As a result, we obtain a tuple $\mathcal{H}$ such that $\mathcal{H}[i]$ is the number of rows of the $i^{\text {th }}$ coloured group. This operation can be seen as the computation of the histogram of the rows of the tables, which is a well-know primitive that can be parallelised very efficiently. In the above example, $\mathcal{H}_{1}=\langle 1,1,2\rangle$, and $\mathcal{H}_{2}=\langle 2,2,2\rangle$. These histograms are also useful to compute the number of rows of the result table, a crucial information when we have to allocate the exact amount of memory to store the result. Each group of output rows has a number of elements equal to the product of the numbers of rows of the corresponding input groups. Hence, the histogram of the result table, namely $\mathcal{H}_{\oplus}$, is computed as the elementwise product ${ }^{10}$ (denoted as $*$ ) of the input histograms. It is easy to verify that $\langle 1,1,2\rangle *\langle 2,2,2\rangle=\langle 2,2,4\rangle$ is the histogram of the result table in Figure 2. The sum of the values of such histogram is the total number of rows of the result table.

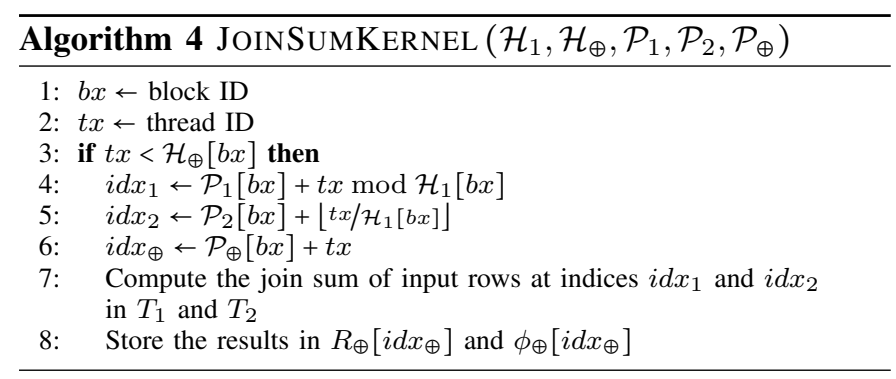

These histograms also allow each thread to efficiently locate its input rows, as well as the index of the output row it is responsible for, by indexing the coloured groups in $T_{1}$ and $T_{2}$. As a first step, we compute the exclusive prefix sum of the input and output histograms, which can be done very efficiently on the GPU [16] and, in our case, it is implemented with the NVIDIA CUB library. Given an histogram $\mathcal{H}$, its exclusive prefix sum $\mathcal{P}$ is a tuple in which each element $\mathcal{P}[i]$ represents the index of the first row of the $i^{\text {th }}$ coloured group. With these data structures, each thread can compute its row in the join sum result. Algorithm 4 represents the actual kernel function executed by the GPU, which receives as inputs the histograms of $T_{1}$ and the output histogram (i.e., $\mathcal{H}_{1}$ and $\mathcal{H}_{\oplus}$ ), ${ }^{11}$ as well as the corresponding prefix sum tuples (i.e., $\mathcal{P}_{1}, \mathcal{P}_{2}, \mathcal{P}_{\oplus}$ ). The variable assignment and the value parts of the output table are respectively denoted as $R_{\oplus}$ and $\phi_{\oplus}$.

It is important to note the absence of divergence in Algorithm 4, thanks to the fact that the only branch instruction (Line 3) is used to limit the number of running threads to the amount needed, i.e., $\mathcal{H}_{\oplus}[b x]$. For the sake of clarity, we made a number of simplifications in Algorithm 4. First, here we

\footnotetext{
${ }^{10}$ Element-wise product is an embarrassingly parallel operation.

${ }^{11}$ We do not explicitly provide $\mathcal{H}_{2}$ to the kernel, since this information is implicitly included in $\mathcal{H}_{1}$ and $\mathcal{H}_{\oplus}$, i.e., $\mathcal{H}_{2}[i]=\mathcal{H}_{\oplus}[i] / \mathcal{H}_{1}[i]$.
}

do not explicitly mention the use of shared memory, which is used to exploit the data reuse ${ }^{12}$ inherent to the join sum operation, so to avoid unnecessary memory accesses to the global memory. The properties of these memory transfers between shared and global memory are discussed in Section V-A. Furthermore, we assume that each coloured group of rows is computed by exactly one block of threads. In contrast, our actual implementation realises a dynamic load balancing by assigning the appropriate number of groups to each block, in order to achieve a higher GPU occupancy and computational throughput. This number is determined by the amount of shared memory of the GPU and the maximum number of threads per block. It is possible that a single group of rows is larger than the available shared memory: this case is managed by splitting such a group into a number of sub-groups, once again with the objective of maximising the GPU occupancy.

\section{Maximisation GPU Computation}

In this section, we describe how we implement the maximisation (which corresponds to the marginalisation operation of BE) on GPUs, exploiting the data layout discussed in Section IV-A. In particular, we adopt the same preprocessing phase detailed by Algorithm 3, with the sole difference that the set of shared variables is represented by all the variables in the scope of the table, excluding the one we want to marginalise. Intuitively, this corresponds to move such a variable to the Most Significant (MS) place. In this case, if we compute the histogram $\mathcal{H}$ and the exclusive prefix sum $\mathcal{P}$ as previously described, we are able to index the groups of rows that must be considered when computing the maximum for the output, denoted by $R_{\max }$ and $\phi_{\max }$ for the variable assignment and the value part. Algorithm 5 implements the maximisation kernel.

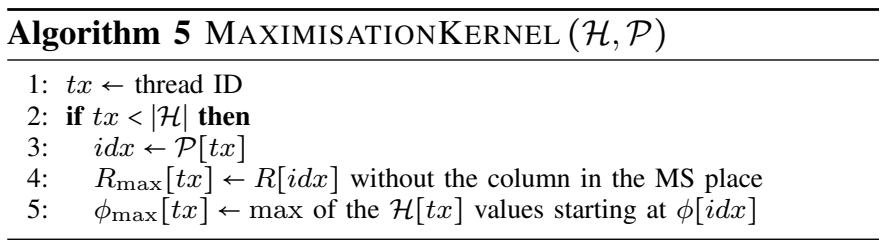

Algorithm 5 shows a simplified version of our actual implementation, in which we generate the appropriate number of threads and blocks on the basis of the size of the input. In contrast with the join sum operation, we do not have any data reuse (i.e., each input row is accessed by exactly one thread), hence the use of shared memory is not necessary. As a final performance remark, notice that the maximisation of the $\mathcal{H}[t x]$ elements at Line 5 is sequentially executed by the thread. Nevertheless, this aspect has a negligible impact of the computational throughput of our approach, since $\mathcal{H}[t x]$ depends on the size of the domain of the marginalised variable ${ }^{2}$ and, in our experiments, it is usually a small value. When the considered variable has a binary domain, Line 5 collapses to one single max operation.

Notice that Algorithms 4 and 5 correctly implement the $\oplus$ and $\Downarrow$ operations of $\mathrm{BE}$, hence preserving its optimality.

\footnotetext{
${ }^{12}$ Blue rows in Figure 2 both refer to the same input row in $T_{1}$, hence both the corresponding threads can reuse the same input data.
} 


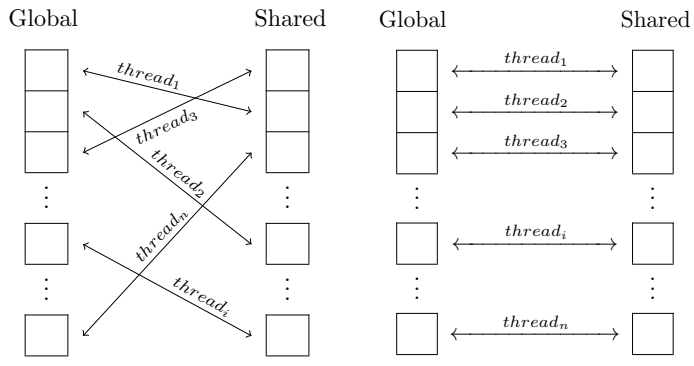

Fig. 8: Uncoalesced vs. coalesced memory accesses.

\section{Data TRANSFERS}

In the following sections we detail how our technique allows an optimised management of data transfers, thanks to full memory coalescence and pipelining.

\section{A. Global-Shared Memory Transfers}

Memory hierarchy in GPUs follows a widely adopted design in modern hardware architectures, in which very fast but smallsize memories (i.e., registers, cache and shared memory), which are designed to assist high-performance computations, are stacked above a slower but larger memory (i.e., global memory), suitable to hold large amounts of rarely accessed data. In particular, shared memory resides on each SM and can deliver 32 bits per two clock cycles. To increase performance, it is mandatory to exploit such a low latency memory to store information that needs to be used very often. On the other hand, accessing global memory is particularly costly (400800 clock cycles), and should be minimised to achieve a good compute-to-memory ratio.

A common programming pattern suggests to split input data into tiles that fit into shared memory (i.e., 48 Kilobytes of information) and to complete all the computational tasks using only such data. This allows to minimise global memory accesses for each kernel execution. Coalesced accesses are the optimal way to carry out such data transfers, which is related to the principle of spatial locality of information.

Memory coalescing refers to combining multiple transfers between global and shared memory into a single transaction, so that every successive 128 bytes (i.e., 32 single precision words) can be accessed by a warp (i.e., 32 consecutive threads) in a single transaction. In general, sparse or misaligned data organisation may result in uncoalesced loads, serialising memory accesses and reducing the performance, while consecutive and properly aligned data chunks enable full memory coalescing (Figure 8 ). Thanks to the previously explained preprocessing phase, the portion of input data needed by each thread block is read from global memory with fully coalesced memory accesses, since such data is already organised in consecutive addresses. The transfers are further optimised using vectorised ${ }^{13}$ memory accesses in order to increase bandwidth, reduce instruction count and improve latency.

\footnotetext{
${ }^{13}$ Vectorised memory instructions compile to single LD.E.128 and ST.E. 128 instructions to transfer chunks of 128 bits at a time.
}

\section{B. Host-Device Data Transfers}

The table layout presented in Sections III and IV allows tables to be split into several data segments and threads to independently operate in each segment. This leads to a twofold improvement: i) we devise a pipelined flow of smaller copyand-compute operations, by amortising the cost of CPU-GPU data transfers on the overall algorithm performance; ii) we can process tables that do not fit into global memory, by breaking them into more manageable chunks. This allows our approach to perform $\mathrm{BE}$ even on problems that were intractable for previous approaches [17], [18].

1) Pipelining: The standard pattern of GPU computation requires the whole input to be transferred to the global memory before starting the kernel execution. The results are then copied back to the host memory. Such synchronous approach can be improved if the kernel can start on a partial set of input data, while the copy process is still running.

Figure 9 shows the proposed pipelined model of computation, in which a single GPU message passing operation has been split into four stages (marked by different colours). Each computation kernel $\mathrm{K}_{i}$ executes as soon as the corresponding input data subset has been transferred by means of $\mathrm{H} \rightarrow \mathrm{D}_{i}$. This solution applies to GPU architectures that feature only one copy engine (i.e., data between host and device can be transferred through a single channel only). Data segments for table processing are necessarily serialised, thus allowing overlapping between one kernel execution and one data transfer only. In our experiments focusing on BP, we found that, in average, this approach achieves a performance improvement of $50 \%$ w.r.t. synchronous data transfers. Most recent and advanced GPUs (e.g., NVIDIA Kepler) feature an additional copy engine, which enables a further degree of parallelism between data transfers and computation. On these architectures, this approach exploits the supplementary channel to overlap input and output data transfers (see Figure 10). Such a fully pipelined model of computation achieves, in average, a performance improvement of $75 \%$ w.r.t. synchronous data transfers, achieved in our experiments on WCSPs.

2) Large Tables Processing: Our technique can be applied to execute BE-based algorithm even when tables do not fit into the GPU global memory, by splitting large tables into smaller data structures. In particular, this division is achieved by computing the maximum number of kernels, namely $\max _{s}$, which can execute at the same time without exceeding the memory capabilities of the device. In our implementation, $\max _{s}$ is dynamically determined at runtime as the maximum number of kernels whose total amount of input and output data can be stored into global memory. In addition, we also take into account the space constraints deriving from the use of shared memory (see Section IV-B), by enforcing that single coloured chunks of data can fit in such memory. Figure 11 shows an example, in which $\max _{s}=2$. Each kernel $\mathrm{K}_{i}$ is enqueued in stream $i$ mod $\max _{s}$. Transaction $\mathrm{H} \rightarrow \mathrm{D}_{3}$ cannot be scheduled in parallel with $\mathrm{D} \rightarrow \mathrm{H}_{2}$ (unlike the example of Figure 10), as it would violate the above mentioned memory constraint. Thus, one time slot is skipped in order to complete the copy $\mathrm{D} \rightarrow \mathrm{H}_{1}$ and to free an adequate amount of memory before starting 


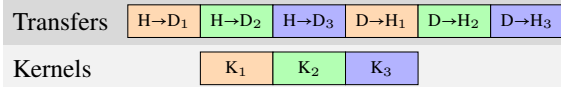

Fig. 9: Asynchronous data transfers.

\begin{tabular}{|c|c|c|c|c|c|}
\hline Host $\rightarrow$ Device & $\mathrm{H} \rightarrow \mathrm{D}_{1}$ & $\mathrm{H} \rightarrow \mathrm{D}_{2}$ & $\mathrm{H} \rightarrow \mathrm{D}_{3}$ & & \\
\hline Kernels & & $\mathrm{K}_{1}$ & $\mathrm{~K}_{2}$ & $\mathrm{~K}_{3}$ & \\
\hline Device $\rightarrow$ Host & & & $\mathrm{D} \rightarrow \mathrm{H}_{1}$ & $\mathrm{D} \rightarrow \mathrm{H}_{2}$ & $\mathrm{D} \rightarrow \mathrm{H}_{3}$ \\
\hline
\end{tabular}

Fig. 10: Full pipeline.

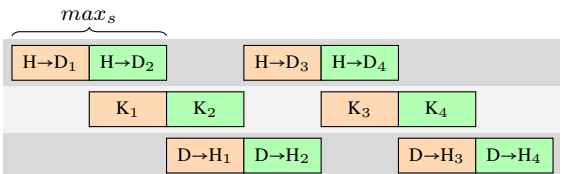

Fig. 11: Limited number of streams.
$H \rightarrow D_{3}$. The serialisation of these two operations is a direct consequence of their execution in the same stream (i.e., stream 1). Even though the hardware constraints limit the size of data to be processed, the proposed approach allows oversized tables to be processed in multiple steps, improving scalability. As an example, 5 out of 7 instances in the considered WCSP dataset (see Section VI-B) cannot be solved without this capability of handling large tables.

\section{EXPERIMENTAL RESULTS}

In order to evaluate appropriately our approach presented in Sections III and IV, we conducted two different set of experiments, discussed respectively in Sections VI-A and VI-B. First, we discuss the results obtained executing BP on JTs (using complete tables), then we test our approach that handles incomplete tables to solve WCSPs. Our approach is implemented in $\mathrm{CUDA}^{14}$ and all our experiments are run on a machine with an AMD A8-7600 processor, 16 GB of memory and an NVIDIA Tesla K40.

\section{A. BP on JTs}

In this section we benchmark the approach described in Section III, i.e., CUDA-BP, which exploits the completeness of tables to achieve a better indexing of potential tables when executing BP on JTs. We compare our approach with the best approach (i.e., the SVR regression model) published by Zheng and Mengshoel [18], using the authors' implementation. We use the same BN dataset, ${ }^{15}$ which comprises various BNs with heterogeneous structures and variable domains. We compile each BN into a JT, which is then used as input for both approaches in order to guarantee a fair comparison. Table I details some features of our JTs, i.e., the number of junction tree nodes resulting from their compilation and the minimum, maximum and average size of the potential and separator tables. Following Zheng and Mengshoel [18], the compilation of these networks into the corresponding junction trees has been done offline, before the execution of the belief propagation algorithm. For this reason, it has been excluded from the runtime measurements.

Table II reports the runtime in milliseconds corresponding to the following phases of the BP on JTs algorithm: i) The total time required to complete all the reduce and scatter phases in the sequential version; ii) The total time required to preprocess all potential tables using our technique; iii) the total time required to complete the data transfers between the host and the device; iv) the total time required to complete all the reduce and scatter phases in our GPU approach; v) our GPU speedup;

\footnotetext{
${ }^{14}$ Available at https://github.com/filippobistaffa/CUBE.

${ }^{15}$ Available at http://bndg.cs.aau.dk/html/bayesian_networks.html.
}

vi) the total time required to complete all the reduce and scatter phases in the GPU approach by Zheng and Mengshoel based on the SVR regression model; vii) Zheng and Mengshoel's speedup. Since the preprocessing phase must be done only once and can be avoided when any new evidence is received and propagated, it has not been considered in the calculation of the speedup. Moreover, we do not consider the runtimes relative to data transfers in such calculation, as such time is amortised thanks to our pipelining technique (Figure 10). For a fair comparison, transfers are also excluded when calculating the speedup for Zheng and Mengshoel's approach.

In our tests, our algorithm outperforms the counterpart in the majority the instances, i.e., all except in the Water network, where runtimes are comparable. In more detail, our approach achieves speedups at least $56 \%$ higher than the counterpart in the Barley dataset (i.e., 33.03× vs $21.14 \times$ ). Our best improvement w.r.t. the counterpart happens on the Mildew network, where our approach runs $39 \times$ faster than the CPU version, and it produces a GPU speedup that is the $466 \%$ higher than the counterpart. In general, our approach produces speedups that increase when the average potential table size increases (see Table I). In fact, we achieve speedups less than $10 \times$ only with small instances (i.e., $\mathrm{Munin}_{2}$ and $\mathrm{Munin}_{3}$ ).

\section{B. WCSPS}

WCSPs involve incomplete tables, as they contain some unfeasible variable assignments. Thus, we apply the approach in Section IV. We consider the SPOT5 dataset [14], a standard dataset that models the problem of managing an Earth observing satellite as a WCSP, to maximise the importance of the captured images, while satisfying some feasibility constraints.

The main objective of these experiments is to evaluate the speedup that can be achieved adopting our parallel approach, which is compared to a sequential $\mathrm{BE}$ version that uses a simple implementation for the join sum and the maximisation operations. Our speedup is compared with the one achieved by the approach by Fioretto, Le, Pontelli, et al. [24] (i.e., the most recent GPU implementation of the BE algorithm) considering the same sequential $\mathrm{BE}$ implementation. The counterpart approach is implemented using the source code provided by the authors.

Table III shows the runtime in seconds (including preprocessing and data transfers) needed to solve the instances of our reference domain by our parallel approach, i.e., CUDABE, compared to its sequential version, i.e., BE. Such table also reports the number of variables and the induced width of these instances. The results show that CUDA-BE provides a speedup of at least 2 orders of magnitude w.r.t. the sequential algorithm, by reaching a maximum of $696.02 \times$. Such speedup increases consistently with the size of the instances (i.e., the 
TABLE I: Bayesian Networks.

\begin{tabular}{crrrrrrrr} 
& Mildew & Diabetes & Barley & Munin $_{1}$ & Munin $_{2}$ & Munin $_{3}$ & Munin $_{4}$ & Water \\
\hline Number of JT nodes & 29 & 337 & 36 & 162 & 854 & 904 & 877 & 21 \\
Max Potential size & 1249280 & 84480 & 7257600 & 38400000 & 151200 & 156800 & 448000 & 589824 \\
Avg Potential size & 117257 & 29157 & 476133 & 516887 & 2400 & 3404 & 10102 & 144205 \\
Min Potential size & 336 & 495 & 216 & 4 & 4 & 4 & 4 & 9 \\
Max Separator size & 62464 & 5280 & 907200 & 2400000 & 6048 & 22400 & 56000 & 147456 \\
Avg Separator size & 3950 & 1698 & 38237 & 58691 & 204 & 528 & 1376 & 28527 \\
Min Separator size & 72 & 16 & 7 & 2 & 2 & 2 & 2
\end{tabular}

TABLE II: BP on JTs Results (time values are in milliseconds).

\begin{tabular}{crrrrrrrr} 
& Mildew & Diabetes & Barley & Munin $_{1}$ & Munin $_{2}$ & Munin $_{3}$ & Munin $_{4}$ & Water \\
\hline CPU R/S & 117 & 219 & 1057 & 6584 & 54 & 109 & 315 & 123 \\
Preprocessing & 28 & 71 & 294 & 2395 & 24 & 37 & 106 & 46 \\
Transfers & 12 & 57 & 110 & 1464 & 16 & 39 & 45 & 15 \\
CUDA-BP R/S & 3 & 14 & 35 & 193 & 14 & 19 & 31 & 12 \\
CUDA-BP Speedup & $39 \times$ & $15.64 \times$ & $33.03 \times$ & $34.11 \times$ & $3.85 \times$ & $5.73 \times$ & $10.16 \times$ & $10.25 \times$ \\
SVR R/S & 17 & 34 & 50 & 648 & 48 & 38 & 71 & 14 \\
SVR Speedup & $6.88 \times$ & $6.44 \times$ & $21.14 \times$ & $10.16 \times$ & $1.12 \times$ & $2.86 \times$ & $4.43 \times$ & $8.78 \times$
\end{tabular}

TABLE III: WCSPs Results (time values are in seconds).

\begin{tabular}{crrrrrrr} 
& \multicolumn{1}{c}{$\mathbf{5 4}$} & \multicolumn{1}{c}{$\mathbf{2 9}$} & $\mathbf{4 0 4}$ & $\mathbf{5 0 3}$ & $\mathbf{4 2 b}$ & $\mathbf{5 0 5 b}$ & $\mathbf{4 0 8 b}$ \\
\hline Variables & 67 & 82 & 100 & 143 & 190 & 240 & 200 \\
Induced Width & 11 & 14 & 19 & 9 & 18 & 16 & 24 \\
BE Runtime & 965.66 & 2656.72 & 7584.12 & 6347.98 & 31637.91 & 53710.41 & 76456.15 \\
CUDA-BE Runtime & 3.01 & 5.36 & 12.40 & 17.46 & 58.42 & 77.17 & 120.79 \\
CUDA-BE Speedup & $321.03 \times$ & $495.38 \times$ & $611.67 \times$ & $363.63 \times$ & $541.55 \times$ & $696.02 \times$ & $632.97 \times$ \\
Fioretto et al. Runtime & 10.32 & 20.75 & 41.12 & 38.96 & - & - & - \\
Fioretto et al. Speedup & $93.57 \times$ & $128.03 \times$ & $184.43 \times$ & $162.93 \times$ & - & - & -
\end{tabular}

induced width and the number of variables), showing that the proposed approach correctly exploits the increased amount of parallelism in bigger tables. In fact, the speedup provided by CUDA-BE monotonically increases in the first three WCSP instances (i.e., 54, 29 and 404), in which both the number of variables and the induced width increase. On the other hand, such speedup decreases in instance 503, which, despite having a larger number of variables, is characterised by a lower induced width. Notice that the induced width has a stronger influence on the complexity of the problem [2]. The ability of our method of handling large tables is crucial in this scenario. In fact, 5 out of 7 instances (i.e., 404, 503, 42b, 505b and 408b) cannot be solved without this feature, as their tables exceed the amount of GPU memory. Finally, notice that our approach outperforms the approach by Fioretto, Le, Pontelli, et al. both in terms of runtime and scalability. On the one hand, our approach is, on average, $3.21 \times$ faster than the counterpart in the solution of the first 4 instances. On the other hand, the counterpart approach cannot handle the 3 biggest instances of the SPOT5 dataset, probably due to the different representation of the tables in memory.

\section{CONCLUSION}

In this article we considered the BE framework and we proposed an efficient and scalable highly-parallel approach that is able to harness the computational power of modern GPUs by means of an appropriate data organisation in memory. The proposed approach applies also to problems involving incomplete tables, i.e., tables that do not contain all variable assignments, as well as problems that do not fit into the global memory of the GPU. Furthermore, it enables pipelined data transfers between host and device, thus further improving performance. Our experimental results show that our approach outperforms the state-of-the-art approach for BP on JTs proposed by Zheng and Mengshoel [18], by obtaining speedups ranging from $+56 \%$ to $+466 \%$. The tests on WCSPs confirmed the ability of our technique to achieve a high computational throughput (reaching a speedup of $696.02 \times$ w.r.t. the CPU version), and proving the importance of its ability to process large tables, a necessary feature to solve these instances.

Future work will look at integrating the proposed GPU techniques in other algorithmic frameworks, such as AND/OR search-based approaches [21], in which Mini-Bucket Elimination heuristics [2] are used to guide the search.

\section{REFERENCES}

[1] S. Dasgupta, C. Papadimitriou, and U. Vazirani, Algorithms. McGraw-Hill, Inc., 2006.

[2] R. Dechter, Constraint processing. Morgan Kaufmann, 2003.

[3] V. Chandru, "Variable elimination in linear constraints," Comput. J., vol. 36, no. 5, pp. 463-472, 1993.

[4] R. Dechter and J. Pearl, "Network-based heuristics for constraint-satisfaction problems," $A I J$, vol. 34 , no. 1, pp. 1-38, 1987.

[5] M. Davis and H. Putnam, "A computing procedure for quantification theory," J. ACM, vol. 7, no. 3, pp. 201$215,1960$.

[6] S. L. Lauritzen and D. J. Spiegelhalter, "Local computations with probabilities on graphical structures and their application to expert systems," J. R. Stat. Soc., vol. 50, no. 2, pp. 157-224, 1988.

[7] U. Bertele and F. Brioschi, Nonserial dynamic programming. Academic Press, Inc., 1972.

[8] A. Petcu, "A class of algorithms for distributed constraint optimization," PhD thesis, EPFL, 2007. 
[9] S. Aji and R. McEliece, "The generalized distributive law," IEEE Trans. Inf. Theory, vol. 46, no. 2, pp. 325343, 2000.

[10] R. Farber, CUDA Application Design and Development. Elsevier, 2011.

[11] S. Balla-Arabe, X. Gao, D. Ginhac, V. Brost, and F. Yang, "Architecture-driven level set optimization: From clustering to subpixel image segmentation," IEEE Trans. Cybern., vol. PP, no. 99, pp. 1-14, 2015.

[12] D. Berjon, G. Gallego, C. Cuevas, F. Moran, and N. Garcia, "Optimal piecewise linear function approximation for gpu-based applications," IEEE Trans. Cybern., vol. PP, no. 99, pp. 1-12, 2015.

[13] Y. Tan and K. Ding, "A survey on gpu-based implementation of swarm intelligence algorithms," IEEE Trans. Cybern., vol. PP, no. 99, pp. 1-14, 2015.

[14] E. Bensana, M. Lemaitre, and G. Verfaillie, "Earth observation satellite management," Constraints, vol. 4, no. 3, pp. 293-299, 1999.

[15] N. Satish, M. Harris, and M. Garland, "Designing efficient sorting algorithms for manycore GPUs," in Proc. IEEE IPDPS, 2009, pp. 1-10.

[16] S. Sengupta, M. Harris, Y. Zhang, and J. D. Owens, "Scan primitives for GPU computing," in Proc. ACM GH, 2007, pp. 97-106.

[17] L. Zheng, O. J. Mengshoel, and J. Chong, "Belief propagation by message passing in junction trees: Computing each message faster using GPU parallelization," in Proc. UAI, 2011, pp. 822-830.

[18] L. Zheng and O. Mengshoel, "Optimizing parallel belief propagation in junction trees using regression," in Proc. SIGKDD, 2013, pp. 757-765.

[19] F. Bistaffa, A. Farinelli, and S. D. Ramchurn, "Sharing rides with friends: A coalition formation algorithm for ridesharing," in Proc. AAAI, 2015, pp. 608-614.

[20] K. Kask, R. Dechter, J. Larrosa, and A. Dechter, "Unifying tree decompositions for reasoning in graphical models," AIJ, vol. 166, no. 1, pp. 165-193, 2005.

[21] N. Flerova, R. Marinescu, and R. Dechter, "Weighted heuristic anytime search: New schemes for optimization over graphical models," Ann. Math. Artif. Intell., pp. 152, 2016.

[22] J. H. Reif, "Depth-first search is inherently sequential," Inform. Process. Lett., vol. 20, no. 5, pp. 229-234, 1985.

[23] Y. Xia and V. K. Prasanna, "Distributed evidence propagation in junction trees on clusters," IEEE Trans. Parallel Distrib. Syst., vol. 23, no. 7, pp. 1169-1177, 2012.

[24] F. Fioretto, T. Le, E. Pontelli, W. Yeoh, and T. Son, "Exploiting gpus in solving (distributed) constraint optimization problems with dynamic programming," in Principles and Practice of Constraint Programming, 2015, pp. 121-139.

[25] D. A. F. Alcantara, Efficient hash tables on the GPU. University of California at Davis, 2011.

[26] F. Bistaffa, A. Farinelli, and N. Bombieri, "Optimising memory management for belief propagation in junction trees using GPGPUs," in Proc. IEEE ICPADS, 2014, pp. 526-533.

[27] P. M. Kogge and H. S. Stone, "A parallel algorithm for the efficient solution of a general class of recurrence equations," in IEEE Trans. Comput., 1973, pp. 786-793.

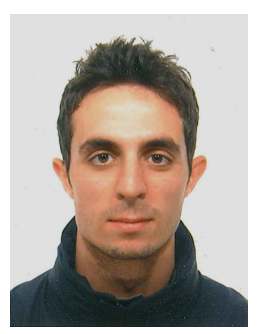

Filippo Bistaffa received the $\mathrm{PhD}$ in Computer Science from the University of Verona in 2016. He is Research Associate at the Dept. of Computer Science of the University of Verona. His research interests comprise combinatorial optimisation problems for realistic applications and GPU computing.

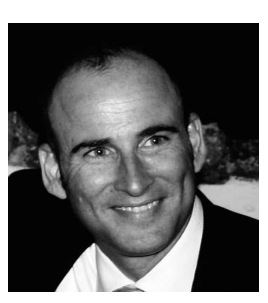

Nicola Bombieri received the $\mathrm{PhD}$ in Computer Science from the University of Verona in 2008 . Since 2008, he is researcher and Professor Assistant at the Dept. of Computer Science of the University of Verona. His research activity focuses on parallel computing, design and verification of embedded systems, and automatic generation and optimization of embedded SW. He has been involved in several research projects and has published more than 70 papers on conference proceedings and journals.

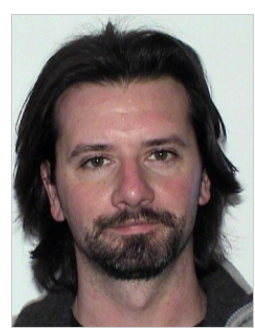

Alessandro Farinelli is Associate Professor at the Dept. of Computer Science of the University of Verona. His research interests comprise theoretical and practical issues related to the development of Artificial Intelligent Systems applied to robotics. 